\title{
Laser ablation for mesial temporal epilepsy: a multi-site, single institutional series
}

\author{
Sanjeet S. Grewal, MD, ${ }^{1}$ Richard S. Zimmerman, MD, ${ }^{2}$ Gregory Worrell, MD, PhD, ${ }^{3,4}$ \\ Benjamin H. Brinkmann, PhD, ${ }^{3,4}$ William O. Tatum DO, ${ }^{5}$ Amy Z. Crepeau, MD, ${ }^{6}$ \\ David A. Woodrum, MD, PhD, ${ }^{7}$ Krzysztof R. Gorny, PhD, ${ }^{7}$ Joel P. Felmlee, PhD, ${ }^{7}$ \\ Robert E. Watson, MD, PhD, ${ }^{7}$ Joseph M. Hoxworth, MD, ${ }^{8}$ Vivek Gupta, MD, ${ }^{9}$ \\ Prasanna Vibhute, MD, ${ }^{9}$ Max R. Trenerry, PhD, ${ }^{10}$ Timothy J. Kaufmann, MD, ${ }^{7}$ \\ W. Richard Marsh, MD, ${ }^{11}$ Robert E. Wharen Jr., MD, ${ }^{1}$ and Jamie J. Van Gompel, MD ${ }^{11}$
}

Departments of ${ }^{1}$ Neurosurgery, ${ }^{5}$ Neurology, and ${ }^{9}$ Radiology, Mayo Clinic, Jacksonville, Florida; Departments of ${ }^{2}$ Neurological Surgery, ${ }^{6}$ Neurology, and ${ }^{8}$ Radiology, Mayo Clinic, Phoenix, Arizona; and Departments of ${ }^{3}$ Neurology, ${ }^{4}$ Biomedical Engineering, ${ }^{7}$ Radiology, ${ }^{10}$ Psychology, and ${ }^{11}$ Neurosurgery, Mayo Clinic, Rochester, Minnesota

OBJECTIVE Although it is still early in its application, laser interstitial thermal therapy (LiTT) has increasingly been employed as a surgical option for patients with mesial temporal lobe epilepsy. This study aimed to describe mesial temporal lobe ablation volumes and seizure outcomes following LiTT across the Mayo Clinic's 3 epilepsy surgery centers.

METHODS This was a multi-site, single-institution, retrospective review of seizure outcomes and ablation volumes following LiTT for medically intractable mesial temporal lobe epilepsy between October 2011 and October 2015. Preablation and post-ablation follow-up volumes of the hippocampus were measured using FreeSurfer, and the volume of ablated tissue was also measured on intraoperative MRI using a supervised spline-based edge detection algorithm. To determine seizure outcomes, results were compared between those patients who were seizure free and those who continued to experience seizures.

RESULTS There were 23 patients who underwent mesial temporal LiTT within the study period. Fifteen patients (65\%) had left-sided procedures. The median follow-up was 34 months (range 12-70 months). The mean ablation volume was $6888 \mathrm{~mm}^{3}$. Median hippocampal ablation was $65 \%$, with a median amygdala ablation of $43 \%$. At last follow-up, 11 $(48 \%)$ of these patients were seizure free. There was no correlation between ablation volume and seizure freedom $(p=$ $0.69)$. There was also no correlation between percent ablation of the amygdala $(p=0.28)$ or hippocampus $(p=0.82)$ and seizure outcomes. Twelve patients underwent formal testing with computational visual fields. Visual field changes were seen in $67 \%$ of patients who underwent testing. Comparing the 5 patients with clinically noticeable visual field deficits to the rest of the cohort showed no significant difference in ablation volume between those patients with visual field deficits and those without $(p=0.94)$. There were 11 patients with follow-up neuropsychological testing. Within this group, verbal learning retention was $76 \%$ in the patients with left-sided procedures and $89 \%$ in those with right-sided procedures.

CONCLUSIONS In this study, there was no significant correlation between the ablation volume after LiTT and seizure outcomes. Visual field deficits were common in formally tested patients, much as in patients treated with open temporal lobectomy. Further studies are required to determine the role of amygdalohippocampal ablation.

https://thejns.org/doi/abs/10.3171/2018.2.JNS171873

KEYWORDS intractable epilepsy; laser interstitial thermal therapy; laser ablation; LiTT; neuropsychology

$\mathrm{T}$ Raditionally, temporal lobectomies, or variants such as selective amygdalohippocampectomy, have been employed as successful treatment options for patients with medically refractory temporal lobe epilepsy. ${ }^{18}$ While laser interstitial thermal therapy (LiTT) has be- come increasingly common as a minimally invasive surgical option for these patients, unlike with temporal lobectomies, there are very limited data in the literature regarding factors associated with seizure outcomes after LiTT., 17,19

Resective surgery has become relatively standard in the

ABBREVIATIONS AVLT = Auditory Verbal Learning Test; BNT = Boston Naming Test; CVLT = California Verbal Learning Test; LiTT = laser interstitial thermal therapy; MPRAGE = magnetization-prepared rapid acquisition gradient echo; MTS = mesial temporal sclerosis.

SUBMITTED August 17, 2017. ACCEPTED February 16, 2018.

INCLUDE WHEN CITING Published online July 6, 2018; DOI: 10.3171/2018.2.JNS171873. 


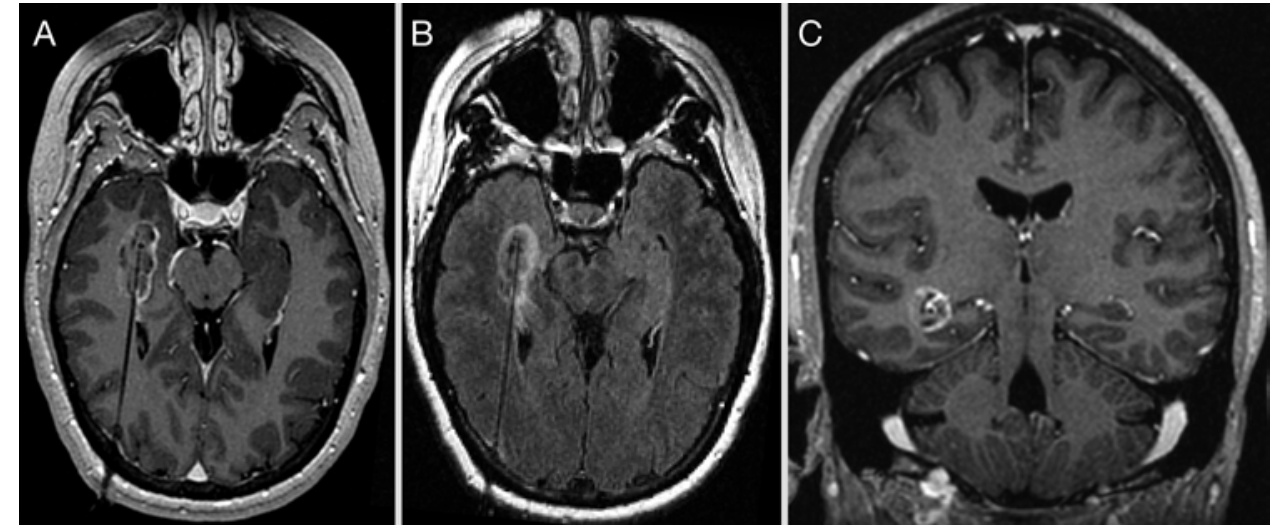

FIG. 1. A: Axial T1-weighted MPRAGE Gd-enhanced image showing a laser cannula in the hippocampus with a zone of nonenhancing ablated tissue surrounded by a thin rim of contrast enhancement. B: Axial T2-weighted FLAIR image showing the expected peripheral zone of T2 hyperintense signal around the area of ablation. C: Coronal T1-weighted MPRAGE Gd-enhanced image showing a peripheral zone of enhancing tissue with the catheter in the center of the zone of enhancement.

amount of amygdala and hippocampus that are removed by the procedure, and LiTT is capable of ablating a cylindrically shaped volume of the amygdala and hippocampus. As ablation volumes are not as large as resection volumes, there is speculation that the volume of ablation correlates with improved seizure outcome, with the assumption that larger ablations would have better seizure outcomes. While there is an emphasis on volume, it is important to note that accuracy may be equally important. Large ablations or resections that eliminate neocortical structures but not the hippocampus are unlikely to be as successful as targeted resections or ablations of the mesial structures. As of now, the optimum volume of hippocampal ablation with LiTT has not been characterized. The primary objective of this study was to test the hypothesis that larger volumes of hippocampal ablation would correlate with better seizure outcomes after LiTT. We further evaluated comorbidities in this cohort, such as visual field and neuropsychological changes following LiTT.

\section{Methods}

This was a multi-site, single-institution, retrospective review of seizure outcomes and ablation volumes following LiTT for medically intractable mesial temporal lobe epilepsy performed between October 2011 and October 2015. This study was approved by the Mayo Clinic institutional review board. The LiTT procedure was performed by 4 different surgeons at the 3 Mayo Clinic epilepsy centers (in Arizona, Florida, and Minnesota). At all sites the same general process was used for targeting the medial temporal lobe. The trajectory started with an occipital approach, avoiding cortical vessels and the ventricle, and targeting the body and head of the hippocampus and as much of the amygdala as possible. Stereotactic guidance included 3 technologies: conventional frame-based (Compass or Leksell), frameless (Stealth) with in-bone fiducial markers, or intraoperative MR-guided placement (Clearpoint). In all cases, multiple overlapping ablations were used.

Pre-ablation volumetric (3D) T1-weighted MR images were acquired in all cases for treatment planning.
The ablations were monitored in real time with MR thermometry. The individual ablations were repeated as the applicator was systematically pulled back until the thermal dose computed in the workstation sufficiently covered the entire targeted volume. Immediately after the ablations, (3D) gadolinium-enhanced T1-weighted images were acquired to delineate tissue damage by gadoliniummediated signal enhancement around the ablation margin (Fig. 1). Pre-ablation and post-ablation volumes of mesial temporal structures (hippocampus + amygdala) in the T1weighted magnetization-prepared rapid acquisition gradient echo (MPRAGE) images were calculated using an automated atlas-based segmentation algorithm (FreeSurfer 4.2) (Fig. 2). The volume of tissue ablated during LiTT was measured in gadolinium-enhanced T1-weighted MR images acquired perioperatively after tissue ablation using a manually supervised spline-based edge detection algorithm (Analyze 12.0, Biomedical Imaging Resource). In all volumetric measurements, hippocampal and amygdala volumes were aggregated, as post-ablation tissue damage made differentiation between amygdala and hippocampus difficult and potentially inaccurate in follow-up imaging. Post-ablation automated segmentations of mesial temporal structures were visually reviewed for accuracy, and if inaccurate, the segmentation was repeated manually with the spline-based edge detection algorithm.

To determine seizure outcomes, results were compared between those patients who were seizure free and those who continued to experience seizures. Engel classification was also used to evaluate seizure outcomes, which were verified by an epileptologist. Visual field assessments were performed postoperatively using standard computational perimetry, with a dedicated ophthalmological consultation.

Neuropsychological testing was performed preoperatively in all cases, and the intent was for all patients to receive postoperative testing. However, only half of the cohort completed postoperative testing. Neuropsychological testing was specific for verbal memory and included the Boston Naming Test (BNT), Auditory Verbal Learning Test (AVLT), and the California Verbal Learning Test 

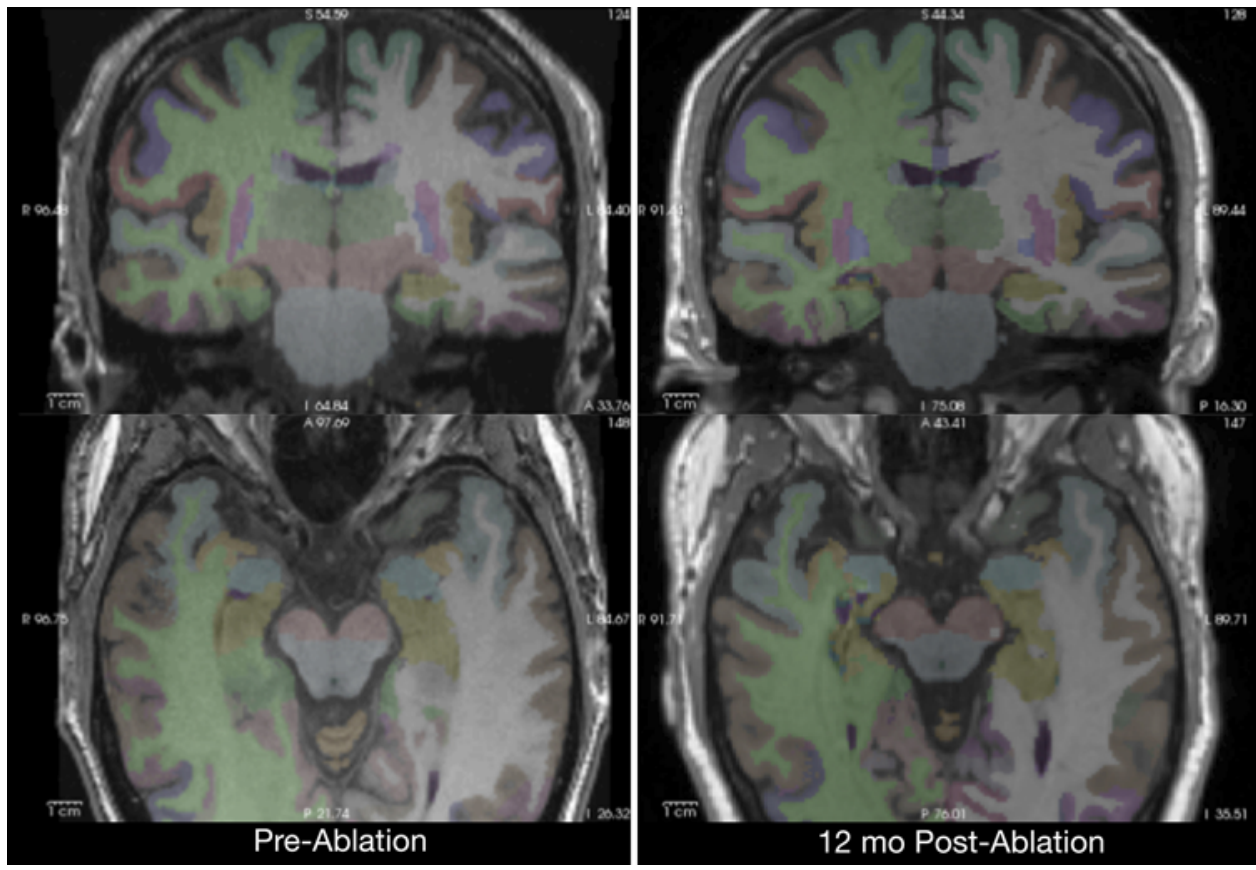

FIG. 2. Coronal and axial T1-weighted pre- and post-LiTT MR images showing segmentation of mesial temporal structures with the FreeSurfer program. These images reveal expected postoperative changes after right mesial temporal lobe ablation. Figure is available in color online only.

(CVLT). All statistical analysis was performed using JMP software (SAS International Inc.) with significance set at $\mathrm{p}<0.05$.

\section{Results}

Twenty-five patients underwent LiTT treatment for mesial temporal lobe epilepsy. In 2 cases, adequate immediate post-ablation imaging was not available, and these patients were excluded from the study. Twelve (52\%) patients were treated at Mayo Clinic Arizona, 6 (26\%) at Mayo Clinic Rochester, and 5 (22\%) at Mayo Clinic Florida. There was no correlation between site and seizure outcomes $(\mathrm{p}=$ $0.66)$. Ten patients $(43 \%)$ were male. The patients' average age was 43.9 years (Table 1). A majority of the patients had ablation for presumed mesial temporal sclerosis (MTS) as determined by MRI (increased T2 signal and hippocampal volume loss); however, 5 patients (22\%) had normal MRI findings, without imaging consistent with MTS. These patients had presumed mesial temporal lobe epilepsy based on clinical seizure semiology and ictal EEG. Pre-ablation mesial temporal volumes are shown in Fig. 3. Most patients had multiple seizure types, but the predominant seizure type for each patient was focal seizures with impaired awareness (formally called complex partial seizures) (74\% of patients) (Table 1). Fifteen patients (65\%) had left-sided procedures. The median follow-up was 34 months (range 12-70 months).

\section{Ablation Volume}

The mean volume of ablated tissue was $6888 \mathrm{~mm}^{3}$ (range 3045.41-11,560.64 $\mathrm{mm}^{3}$ ) (Fig. 4). Median hippocampal ablation was $65 \%$ (range $28 \%-85 \%$ ) with a medi- an amygdala ablation of $43 \%$ (range 1\%-80\%). At last follow-up, $11(48 \%)$ of these patients were seizure free (Table 2). An additional 4 patients (17\%) had Engel Ib outcomes, with no disabling seizures after surgery. Presence of MTS on preoperative imaging was not a predictor of seizure freedom, as 9 of $18(50 \%)$ patients with MTS were seizure free compared to 2 of $5(40 \%)$ patients whose pre-

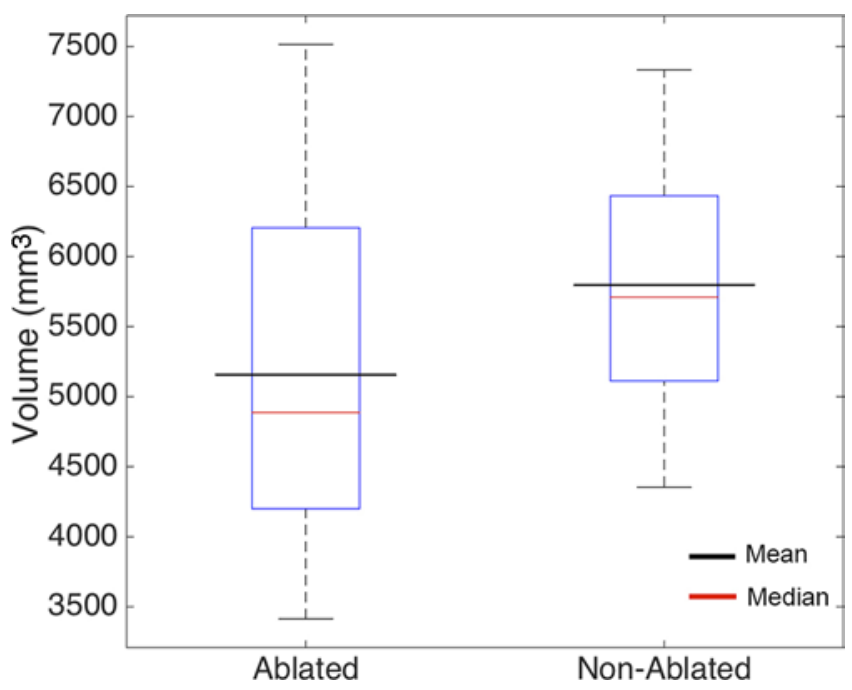

FIG. 3. Mesial temporal volumes measured by FreeSurfer in pre-ablation T1-weighted MPRAGE imaging show lower overall volume on the side undergoing ablation. The majority of patients studied (88\%) had a diagnosis of MTS with volume loss and/or hyperintense signal on FLAIR imaging. Box-and-whisker plots show the 25th and 75th quartiles as demarcated by the box, with the range demarcated by the lines above and below. Figure is available in color online only. 
TABLE 1. Summary of patient demographic and clinical characteristics

\begin{tabular}{|c|c|c|c|}
\hline $\begin{array}{l}\text { Case } \\
\text { No. }\end{array}$ & $\begin{array}{c}\text { Age } \\
\text { (yrs), } \\
\text { Sex }\end{array}$ & Seizures & Pathology \\
\hline 1 & $58, \mathrm{M}$ & Focal seizures w/o impaired awareness & MTS \\
\hline 2 & $57, \mathrm{~F}$ & Focal seizures w/o impaired awareness & MTS \\
\hline 3 & $25, \mathrm{~F}$ & Focal seizures w/ impaired awareness & MTS \\
\hline 4 & $33, \mathrm{~F}$ & Focal seizures w/ impaired awareness & MTS \\
\hline 5 & $42, \mathrm{~F}$ & Focal seizures w/ impaired awareness & MTS \\
\hline 6 & $44, \mathrm{~F}$ & Focal seizures w/o impaired awareness & MTS \\
\hline 7 & $57, \mathrm{~F}$ & Focal seizures w/ impaired awareness & MTS \\
\hline 8 & $46, M$ & Focal seizures w/ impaired awareness & MTS \\
\hline 9 & $48, M$ & Focal seizures w/ impaired awareness & MTS \\
\hline 10 & $52, \mathrm{M}$ & Focal seizures w/o impaired awareness & MTS \\
\hline 11 & $58, \mathrm{M}$ & Focal seizures w/ impaired awareness & Non-lesional \\
\hline 12 & $58, F$ & Focal seizures w/ impaired awareness & MTS \\
\hline 13 & $43, F$ & Focal seizures w/o impaired awareness & MTS \\
\hline 14 & $32, \mathrm{~F}$ & Focal seizures w/ impaired awareness & Non-lesional \\
\hline 15 & $44, \mathrm{M}$ & Focal seizures w/o impaired awareness & Non-lesional \\
\hline 16 & $38, \mathrm{M}$ & Focal seizures w/ impaired awareness & MTS \\
\hline 17 & $42, \mathrm{M}$ & Focal seizures w/ impaired awareness & Non-lesional \\
\hline 18 & $48, F$ & Focal seizures w/ impaired awareness & MTS \\
\hline 19 & $22, \mathrm{M}$ & Focal seizures w/ impaired awareness & MTS \\
\hline 20 & $46, \mathrm{M}$ & Focal seizures w/ impaired awareness & Non-lesional \\
\hline 21 & $20, F$ & Focal seizures w/ impaired awareness & MTS \\
\hline 22 & $60, \mathrm{~F}$ & Focal seizures w/ impaired awareness & MTS \\
\hline 23 & $37, \mathrm{~F}$ & Focal seizures w/ impaired awareness & MTS \\
\hline
\end{tabular}

operative imaging showed no lesion $(\mathrm{p}=0.69)$. There was no correlation between ablation volume and seizure freedom $(p=0.69)$. There was no correlation between Engel class and ablation volume $(\mathrm{p}=0.93)$. There was no correlation between percent ablation of the amygdala $(\mathrm{p}=0.28)$ or hippocampus $(\mathrm{p}=0.82)$ and seizure outcomes. When analysis was restricted to data from the 18 patients with imaging consistent with MTS, ablation volume also did not correlate with seizure outcomes $(\mathrm{p}=0.39)$. Thirteen patients had high-resolution follow-up MRI scans after ablation. These scans were performed an average of 70 days after ablation (range 10-120 days). In this subset of patients, comparison of follow-up MRI studies to the immediate pre-procedure MRI studies revealed an average decline in the volume of the mesial temporal structures of $1095 \mathrm{~mm}^{3}$. Four patients in the cohort had multiple followup MRI examinations, and the longest follow-up obtained was 788 days (Fig. 5). A regression analysis performed on the ablated and contralateral mesial temporal volumes showed an average rate of volume decrease of $3.03 \mathrm{~mm}^{3}$ per day for the ablated mesial temporal structures, while the contralateral mesial temporal volumes remained essentially unchanged (slope $=0.134 \mathrm{~mm}^{3} /$ day).

\section{Visual Outcome}

Twelve patients underwent formal postoperative test-

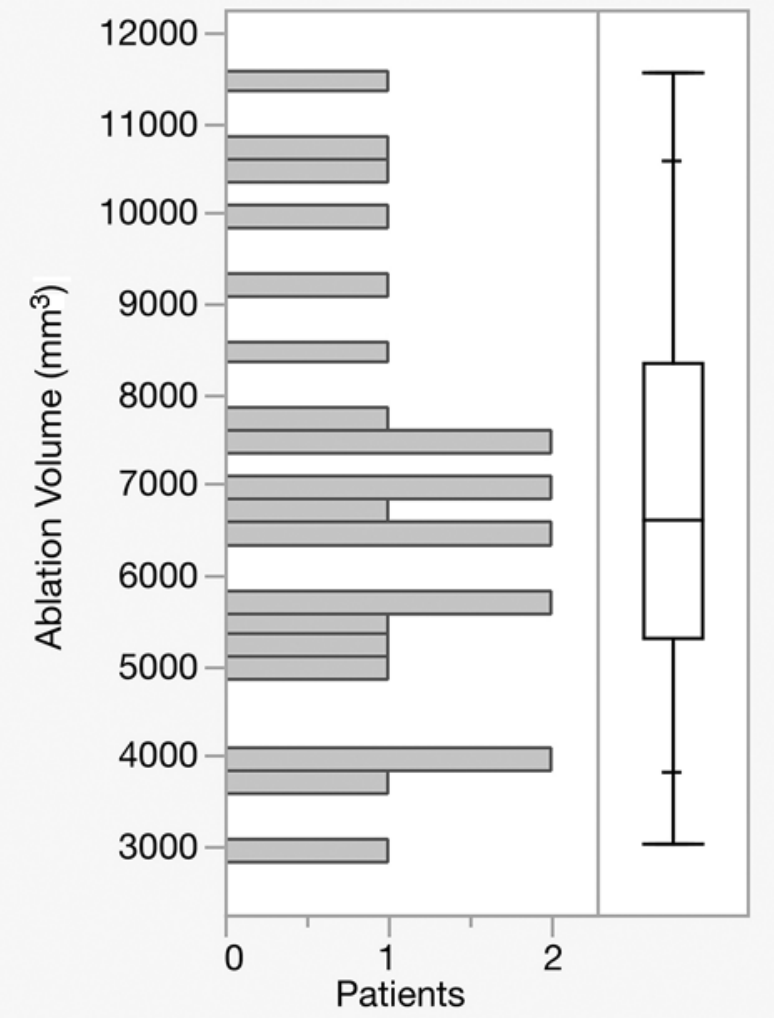

FIG. 4. Left: Bar graph describing the variance in ablation volume across patients. Right: Quartile box plot describing the median ablation volume as well as the 25th and 75th quartiles, with the lines in the whisker plot showing the 10th and 90th quartiles and the whiskers indicating the full range.

ing with computational visual fields. Of these patients, two had near-complete hemianopsias, and 3 patients had quadrantanopsias that they noticed but did not find to be bothersome. Additionally, there were 3 "silent" quadrantanopsias. The remaining 4 patients had normal visual fields. We compared ablation volumes in the 5 patients with clinically noticeable deficits to ablation volumes in the group without clinically noticeable visual field deficits, and there were no statistically significant differences between the 2 groups $\left(6972.27 \mathrm{~mm}^{3}\right.$ vs $6864.57 \mathrm{~mm}^{3}, \mathrm{p}=$ $0.94)$. We repeated this analysis to compare patients with hemianopsias, clinically noticeable quadrantanopsias, silent quadrantanopsias, and no visual field deficits. There was no correlation between ablation volumes and these visual field deficits $(p=0.54)$, or between percentage of hippocampus ablated $(\mathrm{p}=0.96)$ or percentage of amygdala ablated $(p=0.35)$ and visual field deficits. In the 2 patients who developed hemianopsias, further analysis of the lesion was performed. In the first patient, it appears the lesion was too superior and there was thermal damage to the lateral geniculate nucleus (Fig. 6A), while in the second patient, the optic radiations were damaged at their exit from the lateral geniculate nucleus (Fig. 6B).

\section{Neuropsychometric Outcome}

Eleven patients completed preoperative and postop- 
TABLE 2. Ablation side and seizure outcomes

\begin{tabular}{|c|c|c|c|c|}
\hline Case No. & $\begin{array}{l}\text { Ablation } \\
\text { Side }\end{array}$ & $\begin{array}{l}\text { Follow-Up } \\
\text { (mos) }\end{array}$ & $\begin{array}{l}\text { Engle } \\
\text { Class }\end{array}$ & $\begin{array}{l}\text { Seizure } \\
\text { Freedom }\end{array}$ \\
\hline 1 & Right & 70 & la & Yes \\
\hline 2 & Left & 63 & $\mathrm{lb}$ & No \\
\hline 3 & Left & 50 & la & Yes \\
\hline 4 & Left & 52 & $\mathrm{lb}$ & No \\
\hline 5 & Right & 49 & II & No \\
\hline 6 & Right & 48 & III & No \\
\hline 7 & Left & 36 & III & No \\
\hline 8 & Right & 36 & la & Yes \\
\hline 9 & Right & 51 & II & No \\
\hline 10 & Left & 13 & la & Yes \\
\hline 11 & Left & 31 & la & Yes \\
\hline 12 & Left & 31 & $\mathrm{lb}$ & No \\
\hline 13 & Left & 36 & la & Yes \\
\hline 14 & Left & 38 & III & No \\
\hline 15 & Left & 34 & la & Yes \\
\hline 16 & Left & 31 & $\mathrm{lb}$ & No \\
\hline 17 & Left & 29 & II & No \\
\hline 18 & Right & 12 & la & Yes \\
\hline 19 & Left & 21 & la & Yes \\
\hline 20 & Left & 30 & II & No \\
\hline 21 & Right & 18 & II & No \\
\hline 22 & Right & 28 & la & Yes \\
\hline 23 & Left & 22 & la & Yes \\
\hline
\end{tabular}

erative neuropsychological testing. The average time of follow-up for the neuropsychological testing was 7.8 months after LiTT. Of the 11 patients, 7 patients had leftsided procedures. In the group of patients with left-sided procedures, the average verbal learning score (AVLT or CVLT) at follow-up assessment was $76 \%$ of the preoperative score. A smaller decline was noted in the 4 patients with right-sided procedures, with an average verbal learning score of $89 \%$ of the preoperative score. There were 4 patients with declines in their BNT: 2 patients had a decline of 1 point and 2 patients had a decline of 2 points. We consider a decline of 4 points or greater in the BNT to be clinically significant. There was no difference between the sides for those patients with a decline in their BNT as each group had 1 patient with a decline of 2 points and 1 patient with a decline of 1 point.

\section{Discussion}

Penfield reported anterior temporal lobectomy as a method to treat medically refractory temporal lobe epilepsy as early as $1950 .{ }^{11}$ A study by Wiebe et al. in 2001 provided Class I evidence of the superiority of anterior temporal lobectomy when compared to medical management in a randomized, prospective controlled trial. ${ }^{18}$ The present study revealed a 58\% seizure freedom rate at 1 year after the procedure. Other studies have corroborated these results, with a meta-analysis showing a $75 \%$ seizure freedom rate at 1 year. ${ }^{8}$

However, concerns regarding neurocognitive deficits after anterior temporal lobectomy have prompted the desire for more precise procedures, such as selective amygdalohippocampectomy. These selective procedures have led to similar rates of seizure freedom $(67 \%) .{ }^{8}$ There is relatively little data regarding seizure outcomes after LiTT for mesial temporal lobe epilepsy. In patients with at least 6 months of follow-up, the reported rates of seizure freedom are around 54\%.9,19 Our results show a slightly lower rate of seizure freedom when compared to traditional approaches such as anterior temporal lobectomy but are in concordance with the literature on LiTT. However, our study involved a mixed cohort of patients, as not all included patients had mesial temporal lobe sclerosis, and this could partially explain the lower seizure freedom rate as patients without identifiable temporal lobe lesions have lower rates of seizure freedom overall., 3,15

Visual field deficits are among the most commonly reported adverse outcomes after anterior temporal lobectomy, occurring in between $28 \%$ and $52 \%$ of patients. ${ }^{5,14} \mathrm{In}$ fact, in cases in which detailed visual field analysis is performed, nearly all hippocampal resections result in some visual field deficit. ${ }^{16}$ However, many of these deficits are clinically "silent." 10 LiTT theoretically has the potential to decrease the rate of visual field deficits, as it is allows surgeons to traverse the optic radiations less in order to perform an ablation. However, there has not been a study to formally assess for visual field cuts in this cohort. ${ }^{19}$ In our study, formal visual field testing was performed in 12 patients, two of whom had clinically significant visual field deficits. Only 4 patients had normal visual fields, and ablation volumes were not correlative with postoperative visual field deficits. In the 2 patients with hemianopsia, it was caused in one case by damage to the laternal geniculate nucleus, while in the other it was caused by damage to the optic radiations as they exit the lateral geniculate nucleus. We believe that the first case was due to a trajectory that was too high in the hippocampus, leading to damage to the inferolateral aspect of the lateral geniculate nucleus, while the second ablation was carried too far posteriorly, leading to damage of the optic radiations. This warrants further study, with more detailed analysis of volume of ablation with respect to the location of the optic apparatus; however, due to these visual field deficits, we currently aim for a trajectory that leads to the middle or inferior aspect of the hippocampus and complete our ablation once we have reached the level of the lateral mesencephalic sulcus.

There is a relative paucity of data regarding neuropsychological outcomes and LiTT, and there is unfortunately not an agreed-upon standardized battery of tests performed at each center. Similar to results reported in previously published literature, ${ }^{4,6,9}$ in our study, LiTT was associated with a slight decrease in verbal learning with preservation of neocortical functions such as tested by the BNT. Consistent with prior studies, this study showed that decline in verbal memory was more prominent in patients undergoing procedures on their dominant side. ${ }^{6}$ However, a small decline was also noted in verbal memory for patients who underwent procedures on their nondominant hemisphere. 
Grewal et al.
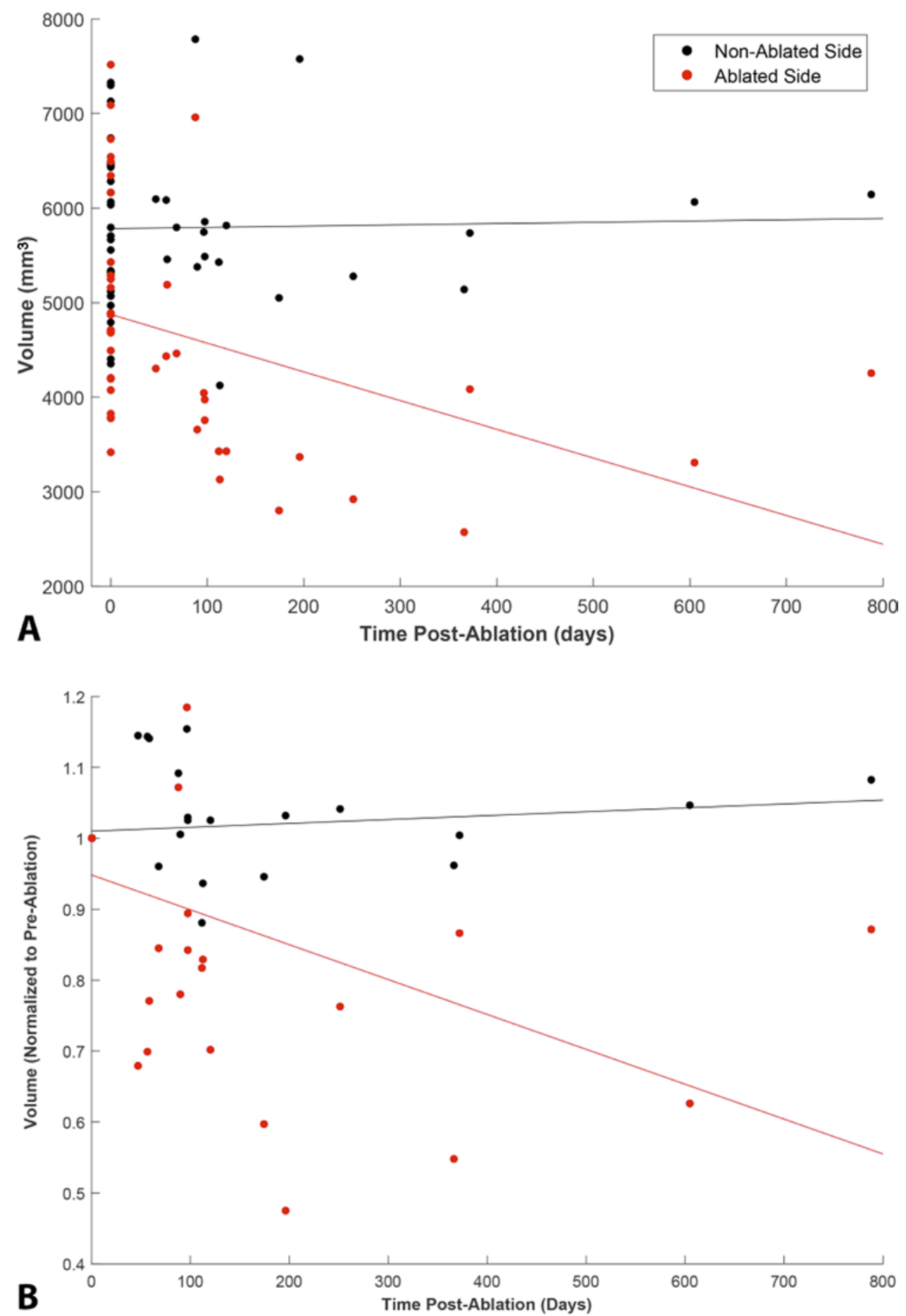

FIG. 5. Mesial temporal volume changes following ablation on the ablated side (red), while the non-ablated side volumes remain constant. A: Volume changes pre- (time $=0)$ and post-ablation as raw $\left(\mathrm{mm}^{3}\right)$ volumes. A regression line fit to the volume measurements has a slope of $-3.03 \mathrm{~mm}^{3} /$ day and a y-intercept of $4875 \mathrm{~mm}^{3}$ for the ablated side, while the non-ablated side's regression has a slope of $0.134 \mathrm{~mm}^{3} /$ day and a y-intercept of $5783 \mathrm{~mm}^{3}$. B: Post-ablation measurements normalized to each patient's preablation volume are plotted. A regression line fit to the normalized measurements has a slope of $-4.9 \times 10^{-4}$ units/day and a yintercept of 0.948 for the ablated side, while the non-ablated side's regression has a slope of $5.5 \times 10^{-5}$ units/day and a y-intercept of 1.01. Figure is available in color online only.

In the surgical literature, there are conflicting data regarding the extent of resection and seizure outcomes in temporal lobe epilepsy. Certain studies have demonstrated that a greater extent of resection leads to improved sei- zure outcomes, ${ }^{2,13}$ while other studies show no correlation between extent of resection and seizure outcomes. ${ }^{1}$ When the specific aspects of resection are analyzed in detail, results suggest that the amount of hippocampal resection is 

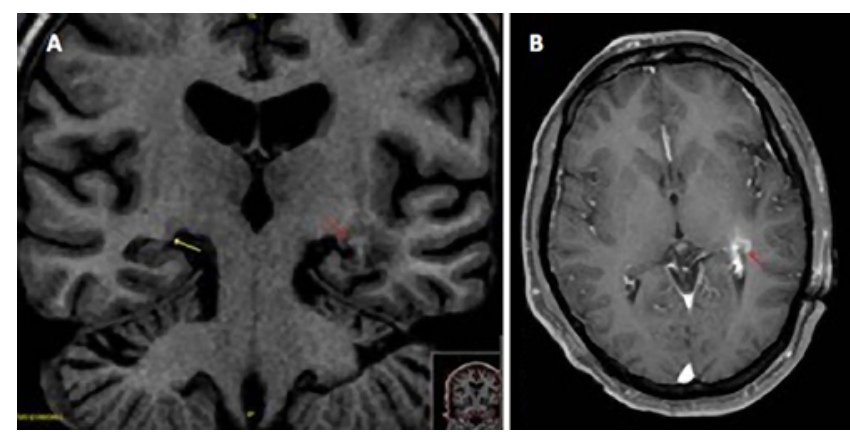

FIG. 6. A: Coronal T1-weighted MR image revealing the location of the lateral geniculate nucleus contralateral to the lesion (yellow arrow); postablation changes are noted in the area of the ipsilateral lateral genticulate nucleus (red arrow). B: Axial Gd-enhanced T1-weighted MR image revealing thermal damage to the optic radiations as they exit the lateral geniculate nucleus (red arrow). Figure is available in color online only.

potentially a better correlate of seizure outcomes. ${ }^{7}$ When analyzing other minimally invasive methods such as radiosurgery, preliminary studies revealed that there was a very tight window of 5.5 to $7.5 \mathrm{ml}$, below which efficacy decreased and above which resulted in excessive toxicity. ${ }^{12}$ In our study, there was no correlation between seizure outcomes and ablation volumes.

One notable point from this retrospective review is the variance in percent ablation of the amygdala (range $1 \%-80 \%$ ). Based upon prior temporal lobectomy results, the surgical goal with LiTT was to achieve as large a hippocampal ablation as possible without causing increased risk to the visual pathways. Due to the curvature of certain hippocampi, a greater degree of ablation of the amygdala was impossible without the addition of a second trajectory. The risk of a secondary trajectory was deemed excessive in these cases, as there were no data to support increased seizure freedom with a greater percentage ablation of the amygdala.

This initial multi-site study, while valuable, is limited due to its retrospective nature as well as its sample size, leading to a low statistical power. This study could also be limited by a narrow range in the variation of ablation volumes, considering that they were all performed by 3 surgeons. This could be improved in a larger multicenter multiple-surgeon trial. Due to the retrospective design of this study, we were unable to assess postoperative visual fields and neuropsychometric testing in the entire cohort, introducing a likely selection bias, as patients who received postoperative testing and had longer follow-up periods could potentially have had these due to poorer outcomes requiring additional care. In addition, while the general techniques were similar across the 3 sites, there was no standardization regarding operative technique or target volumes for ablation.

\section{Conclusions}

This retrospective multi-site study did not show a correlation between seizure outcomes and ablation volumes after LiTT. Two patients had clinically significant visual field deficits, and there was a slight decrease in verbal learning associated with LiTT of the mesial temporal lobe. The results of our study suggest that larger ablations or increased ablation of either the hippocampus or amygdala does not necessarily correlate with seizure outcomes. However, larger ablations were also not correlated with increased complications such as visual field deficits. Future prospective multicenter studies are needed to validate these results in a larger cohort, with further breakdown of the mesial structures, assessing ablation of the subiculum, entorhinal cortex, and uncus as well as assessing postoperative computational visual fields and neuropsychometric testing.

\section{References}

1. Alsaadi TM, Ulmer JL, Mitchell MJ, Morris GL, Swanson SJ, Mueller WM: Magnetic resonance analysis of postsurgical temporal lobectomy. J Neuroimaging 11:243-247, 2001

2. Awad IA, Katz A, Hahn JF, Kong AK, Ahl J, Lüders H: Extent of resection in temporal lobectomy for epilepsy. I. Interobserver analysis and correlation with seizure outcome. Epilepsia 30:756-762, 1989

3. Bell ML, Rao S, So EL, Trenerry M, Kazemi N, Stead SM, et al: Epilepsy surgery outcomes in temporal lobe epilepsy with a normal MRI. Epilepsia 50:2053-2060, 2009

4. Drane DL, Loring DW, Voets NL, Price M, Ojemann JG, Willie JT, et al: Better object recognition and naming outcome with MRI-guided stereotactic laser amygdalohippocampotomy for temporal lobe epilepsy. Epilepsia 56:101113, 2015

5. Egan RA, Shults WT, So N, Burchiel K, Kellogg JX, Salinsky M: Visual field deficits in conventional anterior temporal lobectomy versus amygdalohippocampectomy. Neurology 55:1818-1822, 2000

6. Jermakowicz WJ, Kanner AM, Sur S, Bermudez C, D’Haese PF, Kolcun JPG, et al: Laser thermal ablation for mesiotemporal epilepsy: analysis of ablation volumes and trajectories. Epilepsia 58:801-810, 2017

7. Joo EY, Han HJ, Lee EK, Choi S, Jin JH, Kim JH, et al: Resection extent versus postoperative outcomes of seizure and memory in mesial temporal lobe epilepsy. Seizure 14:541551,2005

8. Josephson CB, Dykeman J, Fiest KM, Liu X, Sadler RM, Jette N, et al: Systematic review and meta-analysis of standard vs selective temporal lobe epilepsy surgery. Neurology 80:1669-1676, 2013

9. Kang JY, Wu C, Tracy J, Lorenzo M, Evans J, Nei M, et al: Laser interstitial thermal therapy for medically intractable mesial temporal lobe epilepsy. Epilepsia 57:325-334, 2016

10. Katz A, Awad IA, Kong AK, Chelune GJ, Naugle RI, Wyllie E, et al: Extent of resection in temporal lobectomy for epilepsy. II. Memory changes and neurologic complications. Epilepsia 30:763-771, 1989

11. Penfield W, Flanigin H: The surgical therapy of temporal lobe seizures. Trans Am Neurol Assoc 51:146-149, 1950

12. Quigg M, Rolston J, Barbaro NM: Radiosurgery for epilepsy: clinical experience and potential antiepileptic mechanisms. Epilepsia 53:7-15, 2012

13. Shamim S, Wiggs E, Heiss J, Sato S, Liew C, Solomon J, et al: Temporal lobectomy: resection volume, neuropsychological effects, and seizure outcome. Epilepsy Behav 16:311314, 2009

14. Tecoma ES, Laxer KD, Barbaro NM, Plant GT: Frequency and characteristics of visual field deficits after surgery for mesial temporal sclerosis. Neurology 43:1235-1238, 1993

15. Téllez-Zenteno JF, Hernández Ronquillo L, Moien-Afshari F, Wiebe S: Surgical outcomes in lesional and non-lesional epi- 
lepsy: a systematic review and meta-analysis. Epilepsy Res 89:310-318, 2010

16. Van Gompel JJ, Welker KM: Visual field mapping to prevent visual field deficits in epilepsy surgery: Seeing the problem. Neurology 83:578-579, 2014

17. Waseem H, Osborn KE, Schoenberg MR, Kelley V, Bozorg A, Cabello D, et al: Laser ablation therapy: an alternative treatment for medically resistant mesial temporal lobe epilepsy after age 50. Epilepsy Behav 51:152-157, 2015

18. Wiebe S, Blume WT, Girvin JP, Eliasziw M: A randomized, controlled trial of surgery for temporal-lobe epilepsy. N Engl J Med 345:311-318, 2001

19. Willie JT, Laxpati NG, Drane DL, Gowda A, Appin C, Hao C, et al: Real-time magnetic resonance-guided stereotactic laser amygdalohippocampotomy for mesial temporal lobe epilepsy. Neurosurgery 74:569-585, 2014

\section{Disclosures}

Dr. Worrell reports direct stock ownership in Cadence Neurosci- ence and NeuroOne Inc. Dr. Kaufmann reports a consultant relationship with SpineThera.

\section{Author Contributions}

Conception and design: Van Gompel, Grewal, Zimmerman, Worrell, Brinkmann, Tatum, Crepeau, Watson, Hoxworth, Kaufmann, Marsh, Wharen. Acquisition of data: Van Gompel, Grewal,

Brinkmann, Gorny, Felmlee, Gupta, Vibhute, Kaufmann, Marsh, Wharen. Analysis and interpretation of data: Van Gompel, Grewal, Trenerry. Drafting the article: Van Gompel, Grewal. Critically revising the article: all authors. Reviewed submitted version of manuscript: all authors. Approved the final version of the manuscript on behalf of all authors: Van Gompel. Statistical analysis: Van Gompel, Grewal, Brinkmann.

\section{Correspondence}

Jamie J. Van Gompel: Mayo Clinic, Rochester, MN. vangompel. jamie@mayo.edu. 